\title{
Pregnane $X$ receptor activation induces FGF19-dependent tumor aggressiveness in humans and mice
}

\author{
Hongwei Wang, ${ }^{1}$ Madhukumar Venkatesh, ${ }^{1}$ Hao Li, ${ }^{1}$ Regina Goetz, ${ }^{2}$ Subhajit Mukherjee, ${ }^{1}$ \\ Arunima Biswas, ${ }^{1}$ Liang Zhu, ${ }^{1}$ Andreas Kaubisch, ${ }^{1}$ Lei Wang, ${ }^{3}$ James Pullman, ${ }^{4}$ Kathleen Whitney, ${ }^{4}$ \\ Makoto Kuro-o, ${ }^{3}$ Andres I. Roig, ${ }^{5}$ Jerry W. Shay, ${ }^{5}$ Moosa Mohammadi, ${ }^{2}$ and Sridhar Mani ${ }^{1}$ \\ ${ }^{1}$ Albert Einstein Cancer Center, Albert Einstein College of Medicine, New York, New York, USA. ${ }^{2}$ Department of Pharmacology, \\ New York University School of Medicine, New York, New York, USA. ${ }^{3}$ Department of Pathology, University of Texas Southwestern Medical Center, \\ Dallas, Texas, USA. ${ }^{4}$ Montefiore Medical Center, Department of Pathology, New York, New York, USA. 5Department of Cell Biology, \\ University of Texas Southwestern Medical Center, Dallas, Texas, USA.
}

The nuclear receptor pregnane $X$ receptor $(P X R)$ is activated by a range of xenochemicals, including chemotherapeutic drugs, and has been suggested to play a role in the development of tumor cell resistance to anticancer drugs. PXR also has been implicated as a regulator of the growth and apoptosis of colon tumors. Here, we have used a xenograft model of colon cancer to define a molecular mechanism that might underlie PXR-driven colon tumor growth and malignancy. Activation of PXR was found to be sufficient to enhance the neoplastic characteristics, including cell growth, invasion, and metastasis, of both human colon tumor cell lines and primary human colon cancer tissue xenografted into immunodeficient mice. Furthermore, we were able to show that this PXR-mediated phenotype required FGF19 signaling. PXR bound to the FGF19 promoter in both human colon tumor cells and "normal" intestinal crypt cells. However, while both cell types proliferated in response to PXR ligands, the FGF19 promoter was activated by PXR only in cancer cells. Taken together, these data indicate that colon cancer growth in the presence of a specific PXR ligand results from tumor-specific induction of FGF19. These observations may lead to improved therapeutic regimens for colon carcinomas.

\section{Introduction}

Decades of clinical research in patients with solid tumors have demonstrated that complete and durable tumor regressions are rare. Indeed the clinical experience with chemotherapy for most solid tumor malignancies indicates that dose-escalation or highdose chemotherapy does not result in an improvement in patient progression-free or overall survival (1-4). The classical theories that explain this apparent lack of benefit posit the existence of drug-resistant tumor stem cells and/or intrinsic and/or induced tumor cell resistance. These cells eventually regrow within the tumor bed or at distant metastatic sites (5-7).

One tumor pathway that may allow for these cells to sense, and respond to, xenochemicals is signaling via the xenobiotic nuclear receptors (e.g., pregnane X receptor/SXR [PXR/SXR], CAR, LXR, farnesol X receptor [FXR], VDR) (8). A receptor in this family, the PXR (also known as SXR, PAR, PRR, or NR1I2), is unique in that it is activated by a diverse array of xenochemicals, including chemotherapies (e.g., paclitaxel, tamoxifen) and naturally occurring endocrine disruptors (e.g., bisphenol A, organochlorine pesticides, methoxychlor, and benzophenone) (9-14). This promiscuous xenosensor plays a central role in regulating liver and gastrointestinal drug metabolism. However, PXR is widely expressed in many different tissues and tumors (e.g., colon, breast, and ovary) (15-19). In search of tissue-specific functions of PXR, we previously demonstrated that PXR activation induces ovarian cancer cell prolif-

Authorship note: Hongwei Wang, Madhukumar Venkatesh, and Hao Li contributed equally to this work.

Conflict of interest: The authors have declared that no conflict of interest exists. Citation for this article: J Clin Invest. 2011;121(8):3220-3232. doi:10.1172/JCI41514 eration and drug resistance (20). While several molecular mechanisms promote this phenotype, recent work indicated that, like the FXR, PXR might also regulate FGF19 expressions in the intestines (21-23). The importance of this observation with regards to cancer is that FGF19 transgenic mice develop hepatocellular carcinoma, and abrogation of FGF19 signaling inhibits colon cancer xenograft growth (24). FGF19, FGF21, and FGF23 comprise the endocrine-acting branch of FGF family, which requires $\alpha / \beta$ klotho coreceptors to activate their cognate FGF receptors (FGFRs). FGF19 signals mainly through FGFR4 in a $\beta$ klotho-dependent fashion and induces multiple intracellular pathways, including MAPK and STATs $(25,26)$. Unlike FGF21 and FGF23, which are strictly coreceptor dependent, FGF19 has also the ability to activate FGFR4 in the absence of $\beta$ klotho. Indeed, FGFR 4 expression has been tied to cancer growth and drug resistance (25, 27-29).

A more controversial area of research is the extent to which environmental xenotoxins play a role in determining human cancer outcomes. Since incurable cancer, which afflicts over 450 persons per 100,000 annually, is a growing health epidemic, it is important to establish a relationship between environmental xenogens (i.e., endocrine disruptors) and cancer (30). Indeed, recent studies on this subject indicate a growing correlation between high exposure to endocrine disruptors (e.g., bisphenol A, xenoestrogens, polycyclic aromatic hydrocarbons) and cancer risk or drug response $(13,31-37)$. The question is particularly relevant given that recent developments in cancer drug therapy (e.g., targeted therapy) are markedly improving survivability. Simultaneously, over the last several decades, our burden of environmental xenotoxins has increased substantially. Recent work implicates several xenogens in cancer cell growth and drug 
A
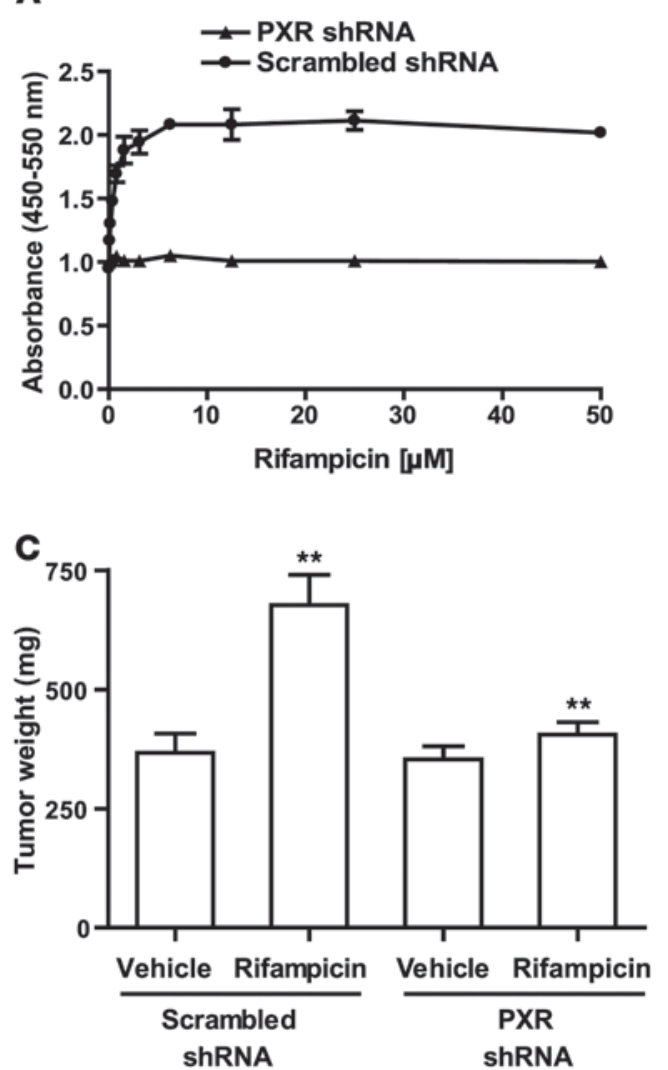

E

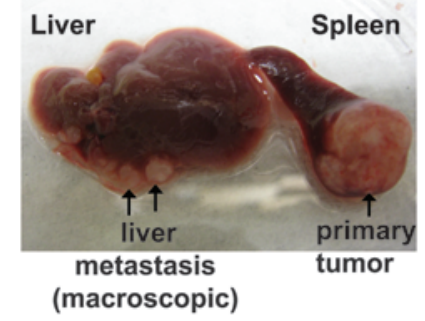

G

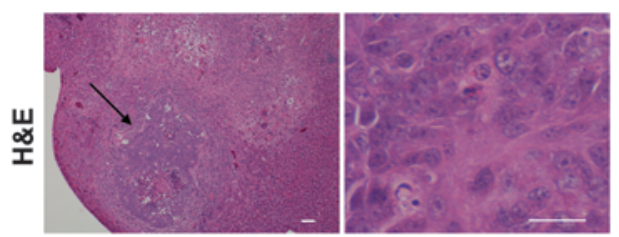

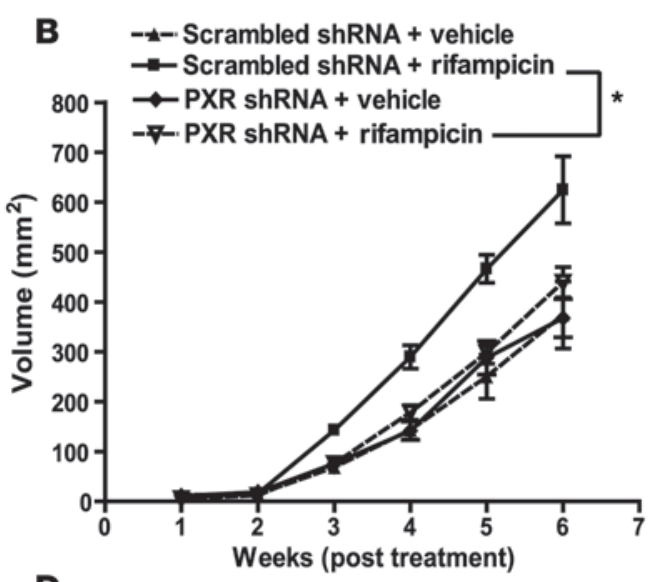

D

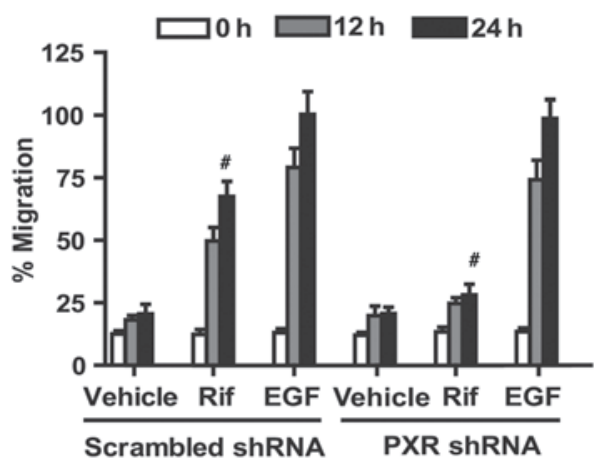

F

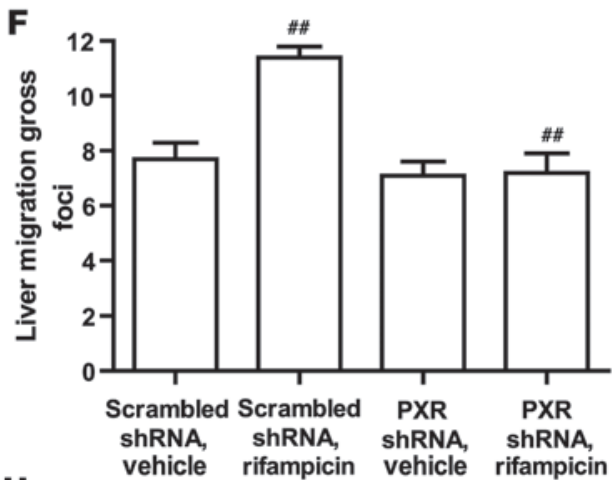

H

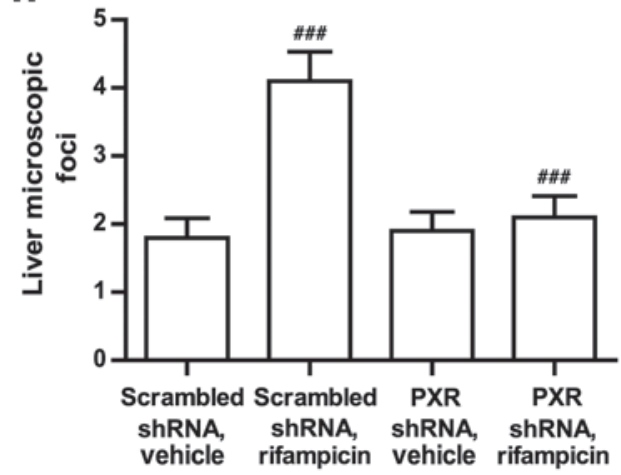

Figure 1

PXR activation induces cell proliferation and cell migration in vitro and in vivo. (A) Proliferation of LS174T cells expressing either scrambled shRNA or PXR shRNA in response to different concentrations of rifampicin (0-50 $\mu \mathrm{M})$. (B) Tumor volumes (C) and tumor weights of xenografts of LS174T cells expressing either PXR shRNA or scrambled shRNA ( $n=12$ per group; day 42). (D) Transwell migration assay performed in the presence or absence of rifampicin $(25 \mu \mathrm{M})$ or EGF (5 nM). (E) Representative photograph of a spleen-liver pair from a single typical mouse sacrificed on day 14 after intrasplenic inoculation with LS174T cells. There is a $100 \%$ metastasis rate to the liver ( $n=8$ mice; data not shown). The black arrows indicate tumor nodules. (F) Histogram depicts mean gross tumor count on the surface of the liver in 48 mice $(n=12$ per group). (G) H\&E stain and microscopic examination (scale bar: $200 \mu \mathrm{m}$ [left panel]; $50 \mu \mathrm{m}$ [right panel]) of tumor nodules in a mouse liver. The black arrow represents a tumor nodule. $(\mathbf{H})$ Histogram depicts mean microscopic tumor count on the surface of the liver obtained from F. (A and $\mathbf{D}) n=4$ in triplicate. DMSO $(0.2 \%)$ was the vehicle for all in vitro experiments. Data are presented as mean \pm SEM. ${ }^{\star} P<0.001,{ }^{* \star} P<0.01,{ }^{\#} P<0.001$, $\# \#<0.001$, \#\#\# $P<0.001$, comparing the 2 groups as indicated. Rif, rifampicin. resistance $(13,31-37)$. Indeed, the molecular pathways governing the tissue-specific phenotypes mediated by chronic exposure to endocrine disruptors are varied, and it is clear that some important effects are mediated via nuclear receptors.
In the current study, we tested the link between PXR-mediated tumor cell growth and the regulation of FGF signaling. Specifically, we show that PXR regulated FGF19 expression in colon cancer cells but not in intestinal crypt cells through direct 
repeat 3-specific (DR3-specific) transcriptional activation of the FGF19 promoter. Strategies to ablate FGF19 signaling prevented PXR-mediated colon tumor growth. Our results have far reaching implications that may mechanistically tie environmental xenogen exposure with cancer drug therapy response and survival.

\section{Results}

PXR activation induces cell proliferation, migration, and metastasis. The ability of PXR ligands to induce hepatomegaly and hepatic regeneration in mice $(38,39)$, as well as ovarian cancer cell proliferation (20), prompted us to investigate whether PXR activation induces a similar phenotype in a human colon tumor cell line (LS174T) and nonneoplastic enterocytes. We have previously reported that PXR is expressed in LS174T cells and that its activation by ligands induces target gene expression (e.g., multidrug resistance-1 [MDR1]) (40). As a control, LS174T cells in which PXR expression was knocked down using shRNA technology were generated and validated to be PXR specific (Supplemental Figures 1 and 2; supplemental material available online with this article; doi:10.1172/ JCI41514DS1). The PXR shRNA phenotype could be rescued by a PXR silent mutant protein, as confirmed by immunoblot analysis (Supplemental Figure 2A), induction of the PXR target gene MDR1 (Supplemental Figure 2B), and PXR-mediated cell growth (Supplemental Figure 2C). To activate human PXR, we used rifampicin, and for activation of mouse PXR, we used pregnenolone$16 \alpha$-carbonitrile (PCN), both prototypical PXR ligands (41-44). Notably, PCN does not activate human PXR, and rifampicin does not activate mouse PXR (45). To examine whether PXR activation in LS174T cells induces proliferation, we measured cell growth rate and survival in response to cell stimulation with rifampicin. Using BrdU labeling to measure cell proliferation, rifampicin treatment increased proliferation of LS174T cells expressing scrambled shRNA by 2.0 to 2.2 fold compared with that in LS174T cells in which PXR expression was knocked down by PXR shRNA expression (Figure 1A). The growth rate constant for rifampicin-treated LS174T cells expressing scrambled shRNA and LS174T cells expressing PXR shRNA was approximately $0.02 \mathrm{~h}^{-1}$ and approximately $0.008 \mathrm{~h}^{-1}$, respectively (Supplemental Figure 3A). Likewise, PXR activation led to increased proliferation of other colon cancer cell lines, namely LoVo (1.5- to 1.6-fold increase; Supplemental Figure 3B) and HCT116 (1.5- to 1.8-fold increase; Supplemental Figure $3 \mathrm{C}$ ). To validate these observations in vivo, we injected LS174T cells into the flanks of athymic nu/nu mice, and, at clinical evidence of tumor growth $\left(\sim 25 \mathrm{~mm}^{3}\right)$, we treated the mice with either rifampicin or vehicle. As shown in Figure 1B, the size of tumors in rifampicin-treated mice at or after day 21 after tumor cell implantation was significantly greater than that of the tumors grown in vehicle-treated mice. Likewise, the tumor weight was significantly greater in rifampicin-treated mice compared with that in vehicle-treated mice (Figure 1C). The effects of rifampicin on tumor growth in vivo were not seen in mice that had been injected with LS174T cells in which PXR expression was knocked down (Figure 1, B and C, and Supplemental Figure 3D).

To assess whether PXR activation also promotes tumor invasiveness, another hallmark of malignancy, we examined LS174T cell migration in response to rifampicin. Cell stimulation with DMSO (vehicle for rifampicin) and EGF served as a negative and positive control, respectively (46). As shown in Figure 1D, rifampicin increased the migration potential of LS174T cells. The migration effect was time dependent and reached its maximum after 24 hours of cell stimulation (migration after 48 hours of cell stimulation was similar; data not shown). PXR knockdown nearly completely abolished the effect of rifampicin on cell migration (Figure 1D). These data show that PXR activation stimulates colon cancer cell migration, suggesting that PXR activation can enhance tumor invasiveness.

To validate the effects of rifampicin on cell migration in vivo, we used an established mouse model of liver metastasis $(47,48)$. In this model, LS174T cells were injected into the spleen, and the effects of rifampicin on liver metastasis development were examined (Figure 1, E and G). Mice were randomized and allocated to receive either LS174T cells expressing PXR shRNA or LS174T cells expressing scrambled shRNA. As shown in Figure 1, F and H, 2-week treatment with rifampicin led to a significant $(-30 \%-40 \%)$ increase in both gross and microscopic metastatic foci in the liver of mice into which LS174T cells expressing scrambled shRNA had been implanted. These effects of rifampicin on metastasis development were not observed in mice inoculated with LS174T cells expressing PXR shRNA (Figure 1, F and H). As expected, PCN, a ligand specific for murine PXR, had no effect on LS174T cell metastasis in vivo (data not shown). Notably, the number of cells (nuclei), either from the primary splenic implant or from the liver metastasis, staining for Ki-67 protein was significantly increased after 2 -week treatment with rifampicin (Supplemental Figure 4). In spleen and liver tissues from mice inoculated with LS174T PXR shRNA cells, the effect of rifampicin on cell proliferation was not observed (Supplemental Figure 4). These data establish that PXR activation enhances LS174T tumor proliferation and metastasis in vivo.

Induction of FGF19 expression by PXR activation mediates proliferation and migration of colon cancer cells. We next explored the mechanisms underlying the effects of PXR activation on proliferation and migration of colon cancer cells. Wistuba and colleagues have previously shown that endobiotic as well as xenobiotic ligands of PXR induce FGF19 gene expression in the LS174T colon cancer cell line $(21,22)$. Based on this finding, one possible mechanism by which PXR activation might mediate cell proliferation and migration is induction of FGF19 expression. To test this, we first examined the effect of rifampicin on FGF19 mRNA expression in LS174T cells. MDR1 (an established PXR target gene in LS174T cells) mRNA expression served as a positive control. As shown in Figure 2A, rifampicin induced FGF19 mRNA expression by approximately 7.4 fold compared with that of vehicle-treated scrambled shRNA cells. Rifampicintreated PXR shRNA cells had significantly lower FGF19 mRNA levels when compared with those of scrambled shRNA treated cells ( $\sim 2.9$ fold vs. $\sim 7.4$ fold, respectively; $P<0.001)$. Consistent with this finding, rifampicin caused an increase in FGF19 protein expression in scrambled shRNA cells that was not observed in PXR shRNA cells (Figure 2B). We next screened 4 additional human colon cancer cell lines for PXR and FGF19 mRNA expression. As shown in Supplemental Figure 5A, the PXR expression levels closely correlated with FGF19 expression. The addition of rifampicin increased PXR activation in every cell line tested and led to corresponding increases in FGF19 expression, further supporting a fundamental relationship between PXR activation and FGF19 expression in colon cancer. Notably, rifampicin had no effect on the expression of FGF23, another member of the FGF19 subfamily (Supplemental Figure 5B), confirming the specificity of its action on FGF19. 

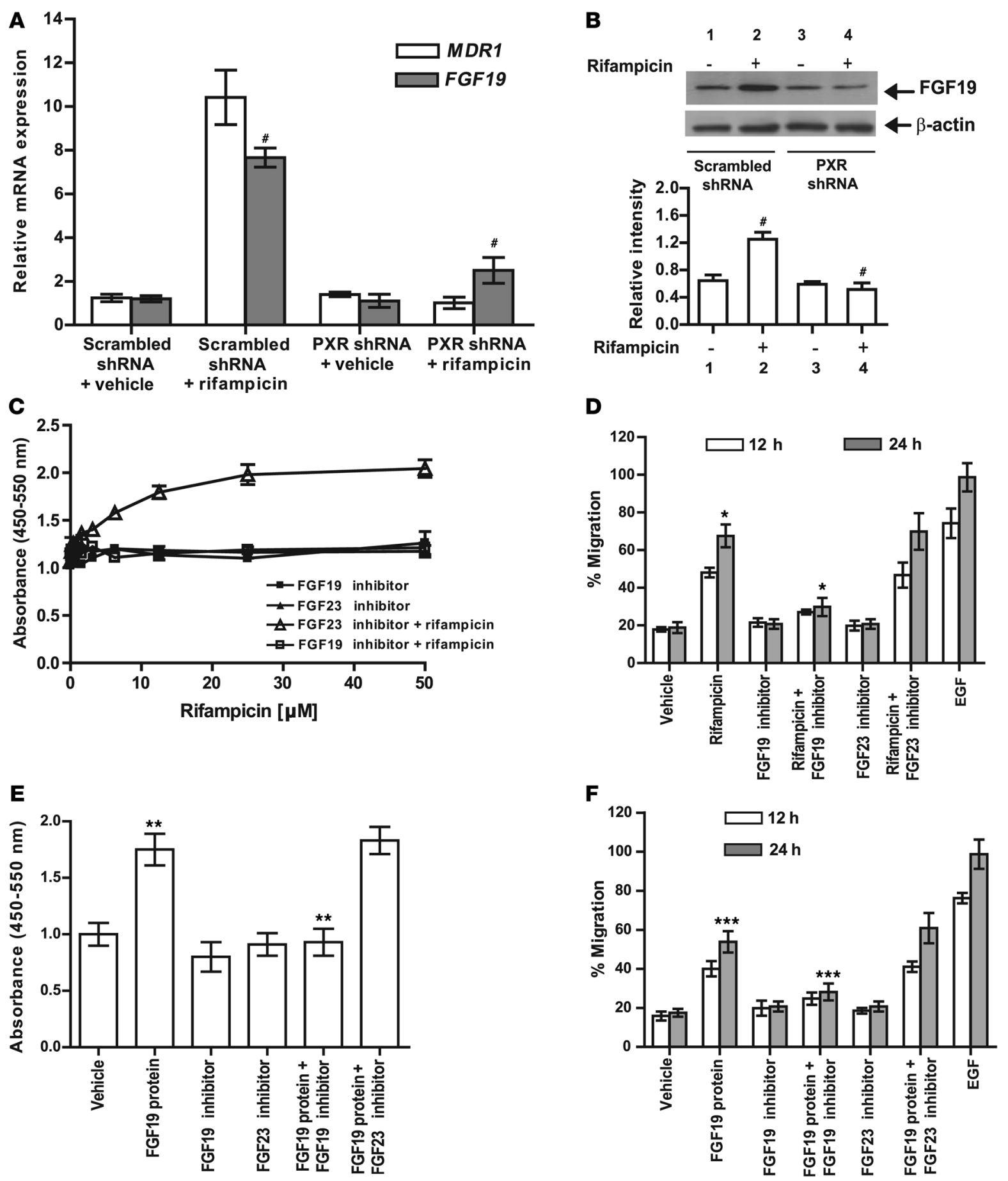

Figure 2

LS174T cell proliferation and migration in response to PXR activation are mediated by induction of FGF19 expression. (A) Real-time quantitative PCR (QPCR) of FGF19 and MDR1 in LS174T cells expressing either scrambled shRNA or PXR shRNA (stimulated with either rifampicin $[10 \mu \mathrm{M}]$ or vehicle). Gene expression changes were calculated using comparative Ct method, with $\beta$-actin as the reference gene and scrambled shRNA plus vehicle as the calibrator. (B) Representative immunoblot analysis of FGF19 from LS174T cells (as in A) exposed to rifampicin $(10 \mu \mathrm{M})$ or vehicle, with $\beta$-actin as loading control. Absolute band intensity (ImageJ; http://rsbweb.nih.gov/ij/) is plotted as a function of lanes from immunoblots. (C) Proliferation of LS174T cells exposed to rifampicin (0-50 $\mu \mathrm{M})$, FGF23 inhibitor (400 ng/ml), and FGF19 inhibitor $(400 \mathrm{ng} / \mathrm{ml})$ as illustrated. (D) Transwell migration assay in the presence or absence of rifampicin $(25 \mu \mathrm{M})$ or vehicle alone or in combination with FGF19 inhibitor (400 ng/ml) or FGF23 inhibitor $(400 \mathrm{ng} / \mathrm{ml})$. (E) Proliferation of LS174T cells that had been stimulated with FGF19 protein $(1,000 \mathrm{ng} / \mathrm{ml})$, with or without FGF19 inhibitor $(400 \mathrm{ng} / \mathrm{ml})$ or FGF23 inhibitor $(400 \mathrm{ng} / \mathrm{ml})$. (F) Transwell migration assay performed with LS174T cells stimulated with rifampicin $(25 \mu \mathrm{M})$ or vehicle alone or in combination with FGF19 inhibitor $(400 \mathrm{ng} / \mathrm{ml})$ and FGF23 inhibitor $(400 \mathrm{ng} / \mathrm{ml})$ as illustrated. Data are presented as mean \pm SEM. (A and $\mathbf{C}-\mathbf{F}) n=4$ in triplicate; (B) $n=3$. DMSO $(0.2 \%)$ was the vehicle for all in vitro experiments. ${ }^{*} P<0.001,{ }^{\star} P<0.001,{ }^{* \star} P<0.001,{ }^{* *} P<0.001$. 
To further test the hypothesis that proliferation and migration of colon cancer cells in response to PXR activation was mediated by induction of FGF19 expression, we examined whether inhibition of FGF19 signaling by an antagonist can suppress the effects of rifampicin on proliferation and migration of LS174T cells. An FGF23 antagonist (49) served as a negative control, since FGF23 is not induced by PXR activation in LS174T cells (Supplemental Figure 5B), and the FGF23 antagonist does not alter cell proliferation or migration (Figure 2, C-F). As shown in Figure 2C, the FGF19 inhibitor abrogated the rifampicin-induced increase in proliferation of LS174T cells, indicating that FGF19 mediates the proliferative activity of rifampicin on LS174T cells. Similarly, the effect of rifampicin on LS174T cell migration was nearly completely abolished by the FGF19 inhibitor (Figure 2D; mean percentage migration, $\sim 73 \%$ in rifampicin-treated LS174T cells vs. $\sim 34 \%$ in cells costimulated with rifampicin and FGF19 inhibitor; $P<0.001$ ), suggesting that induction of FGF19 expression also accounts for cell migration in response to rifampicin. As expected, the FGF23 antagonist had no effect on either proliferation or migration of LS174T cells in response to rifampicin, confirming that the effects of PXR activation are specifically mediated through FGF19.

Based on these data, we reasoned that exogenous FGF19 ligand should induce similar effects on colon cancer cells as observed with the PXR agonist, rifampicin. To test this, we stimulated LS174T cells with recombinant FGF19 protein at concentrations approximately matching those of FGF19 protein secreted by LS174T cells in response to rifampicin (Supplemental Figure 6). Cells were stimulated with FGF19 alone or FGF19 plus FGF19 inhibitor or vehicle. As shown in Figure 2, E and F, the increase in cell survival and migration in response to exogenously added FGF19 ( 1.7to 1.9 -fold increase in cell proliferation) was similar to the effect observed upon cell stimulation with rifampicin ( 2.0- to 2.2-fold increase in cell proliferation; Figure 1A). As expected, the FGF19 inhibitor nearly completely suppressed the effects of the FGF19 protein (Figure 2, E and F; $P<0.001$ ). In a parallel experiment, LS174T cells expressing either scrambled shRNA or PXR shRNA were also treated with FGF19 or vehicle. As shown in Supplemental Figure 7A, the increase in cell survival in response to exogenously added FGF19 was similar regardless of cell PXR content, suggesting that FGF19 alone can initiate growth. This is consistent with a model whereby FGF19 acts downstream of PXR to promote cellular proliferation. We obtained similar data for other colon cell lines (Supplemental Figure 7, B and C). Together, these data support the concept that cell proliferation and migration in response to PXR activation are mediated by induction of FGF19 expression.

FGF19 is a direct target gene of PXR and induces proliferation of primary human colon cancer tissue in vivo. To confirm that FGF19 has tumor growth-promoting activity in vivo, we generated nude mice heterotransplanted with a randomly selected sample of primary human colon cancer tissue. We first passaged 1 human tumor serially through 2 generations of mice and established colonies of similarly aged mice carrying the same heterotransplanted human tumor. These mice were then randomly divided into 8 groups, each receiving one of the following treatments: (a) control antibody, (b) control peptide, (c) rifampicin plus control antibody, (d) rifampicin plus control peptide, (e) rifampicin plus FGF19-neutralizing antibody (1A6; ref. 24), (f) FGF19 protein plus control antibody, (g) FGF19 protein plus FGF19 antibody, and (h) FGF19 antibody plus control peptide. One week after commencing treatment, tumor volumes were measured, and monitoring of tumor growth was continued for another 5 weeks. In agreement with the data shown in Figure 1, mice treated with rifampicin developed larger tumors compared with mice administered control antibody or control peptide (Figure 3A; $P<0.02$ ). The growth advantage of rifampicin-treated tumors in mice was significantly reduced by concomitant treatment with the FGF19 antibody (Figure 3A; $P<0.01)$. Similar to mice treated with rifampicin, mice that had received FGF19 protein developed larger tumors than mice that had been administered control protein (Figure 3A). Cotreatment with the FGF19 antibody 1 A6 nearly completely suppressed the effect of FGF19 protein on tumor growth (Figure 3A). Importantly, mice treated with the $1 \mathrm{~A} 6$ antibody alone exhibited significantly decreased tumor growth compared with that of mice treated with control antibody (Figure 3A), suggesting that FGF19 secreted by the implanted human colon tumor contributed to tumor growth. Together, the data demonstrate that FGF19 promotes growth of colon cancer in vivo.

To further validate that FGF19 alone has growth-promoting properties in vitro and in vivo, we established a lentiviral-based stable expression FGF19 shRNA (see Supplemental Methods and Supplemental Figure 8, A-C). Knockdown of FGF19 decreased growth of LS174T cells (Supplemental Figure 8D; $P<0.001$ ), and this effect could be rescued with exogenous addition of FGF19 protein. Importantly, rifampicin had no significant proliferative effect on FGF19 shRNA cells (Supplemental Figure 8E; $P=0.2$ ). To verify in vivo that FGF19 alone has growth-promoting activity, we implanted LS174T cells expressing either FGF19 shRNA or control shRNA into mice and examined tumor growth from 4 to 9 weeks after implantation. Consistent with our in vitro findings, xenografts of LS174T cells in which FGF19 expression was knocked down exhibited reduced growth compared with that of xenografts of cells expressing control shRNA (Supplemental Figure 8, F and G). Furthermore, rifampicin-induced growth of LS174T xenografts was significantly blunted in FGF19 shRNA tumors compared with that in control shRNA tumors (Supplemental Figure 8G; $P<0.02$ ).

We collected 12 sequential colon cancer tissues from primary colonic surgery performed at Montefiore Medical Center and found by immunohistochemical analysis that 11 out of $12(\sim 92 \%)$ tumors express PXR in variable abundance (data not shown). To determine whether histologic and marker expression of the tumors were preserved on serial passage in mice, we first performed immunostaining of the tumors for PXR and carcinoembryonic antigen (CEA). In addition, H\&E stains were done to assess histology of gland formation. The original histology showed a moderately differentiated colonic adenocarcinoma with prominent mucinous/colloid features. Serially passaged tumors retained this histology. The CEA stain was also similar in distribution both in the original tumor and in serially passaged tumors. Finally, PXR staining was mostly observed as nuclear punctate stains, both in the original tumor tissue and in serially passaged tumors (Figure 3B). These tumors expressed PXR protein and, upon rifampicin activation, induced FGF19 (Figure 3C) to a degree corresponding to the expression levels of PXR mRNA (Figure 3D). There was a clear correlation $\left(r^{2}=0.987\right)$ between $P X R$ mRNA expression levels and FGF19 mRNA expression after activation with rifampicin in vivo (Figure 3D).

Activation by rifampicin of FGF19 signaling in these colon tumor heterotransplants was examined using an electrochemiluminescence-based assay (see Supplemental Methods). Rifampicin induced phosphorylation of FGFR substrate $2 \alpha$ (FRS2 $\alpha$ ), leading 
A
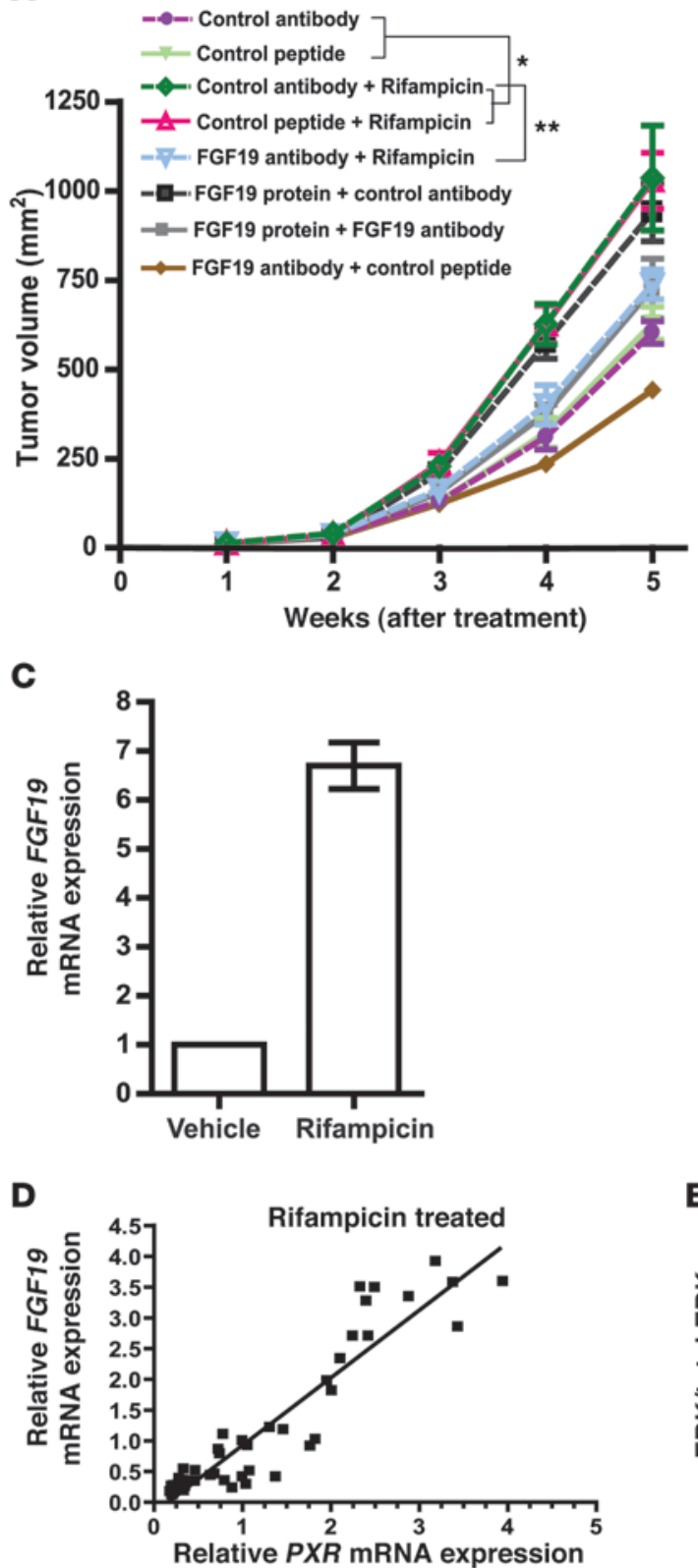

B
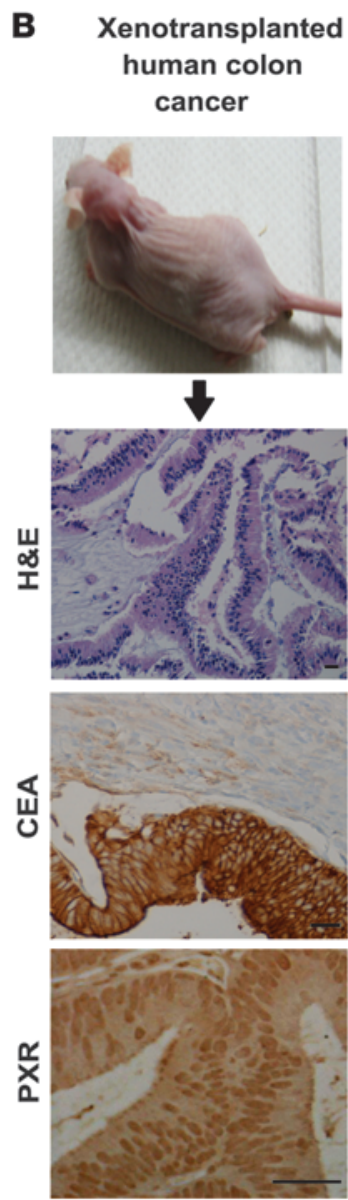

E

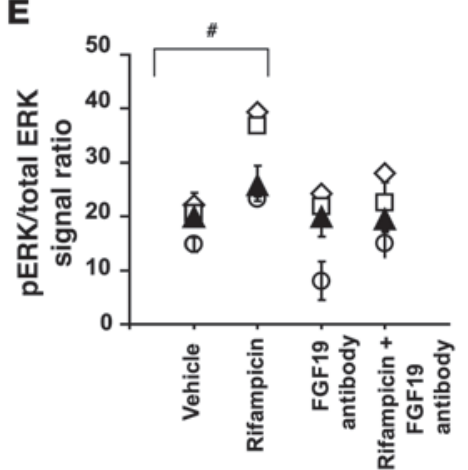

Serially passaged xenotransplanted human colon cancer
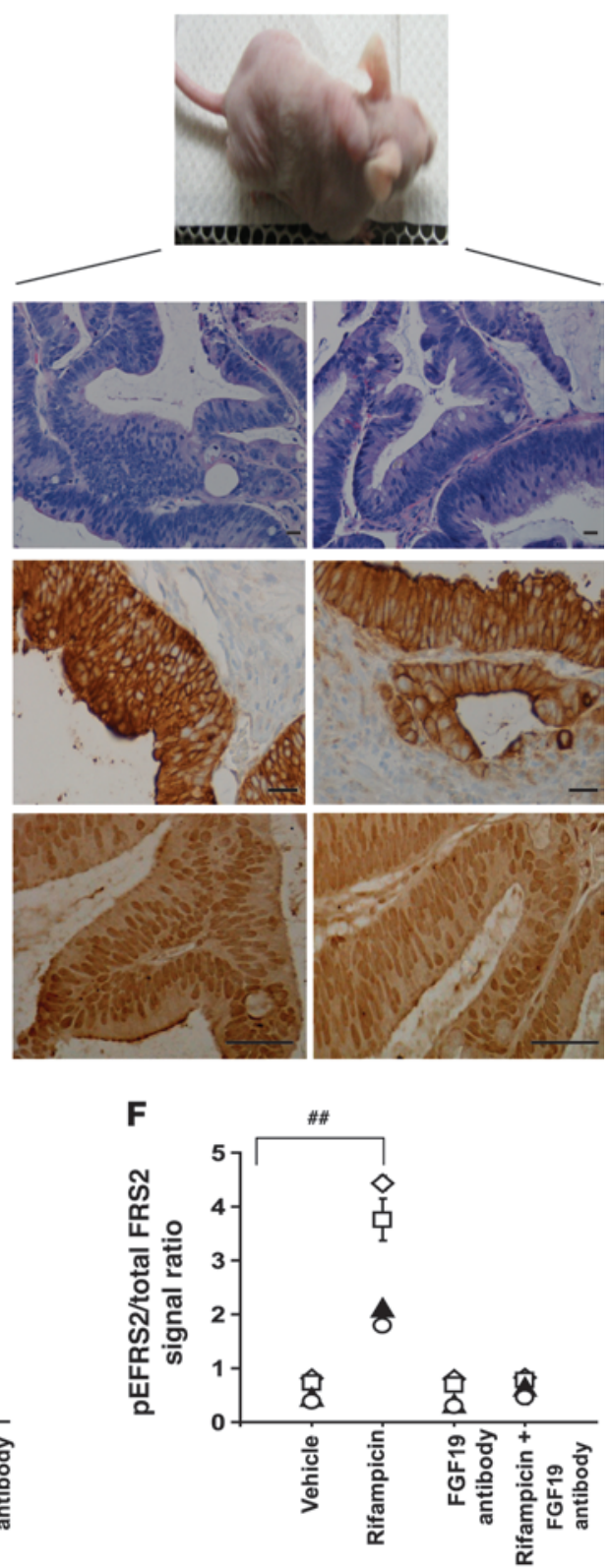

Figure 3

PXR activation in colon cancer induces tumor growth that is inhibited by FGF19 inhibitory antibody. (A) Tumor volumes after treatment of human colon tumor xenotransplants ( $n=12 /$ group) as illustrated. (B) Serially passaged human colon tumor stained with H\&E and antibodies detecting CEA and PXR. Scale bar: $100 \mu \mathrm{m}$. (C) Real-time QPCR for FGF19 in vehicle-treated (30\% polyethylene glycol) $(n=3)$ and rifampicin-treated $(n=3)$ human xenotransplanted tumors ( $n=2$ with 4 replicates each using the same pooled samples of human tumors). (A and C) Data are presented as mean \pm SEM. ${ }^{*} P<0.02,{ }^{* *} P<0.01$, comparing the 2 groups as indicated. (D) Twice passaged primary human tumors were treated with vehicle or rifampicin ( $n=24$ per treatment group). The relative FGF19 and PXR mRNA expression was obtained from values normalized to human $\beta$-actin. The plot shows FGF19 mRNA levels as a function of $P X R$ mRNA levels determined for the same tumor sample $\left(n=48 ; r^{2}=0.987\right)$ treated with rifampicin. ( $\mathbf{E}$ and $\mathbf{F})$ Human colon tumors ( $n=12 /$ group) were treated with vehicle or rifampicin and/or FGF19 antibody, and tumor tissue lysate $(n=3)$ was used for pERK/ERK and pFRS2/FRS2 electrochemiluminescence assay. Data are presented as mean $( \pm$ SD) ratio of signals from all wells per treatment group of phospho-protein/total protein. The signal was plotted as a function of lysate concentrations $(0.75 \mu \mathrm{g}$, white circle; $1.25 \mu \mathrm{g}$, black triangle; $2.5 \mu \mathrm{g}$, white square; $5.0 \mu \mathrm{g}$ : white diamond) used in the assay $\left(n=4\right.$ in triplicate) ${ }^{\#} P<0.05$, \#\# $P<0.05$. 
A

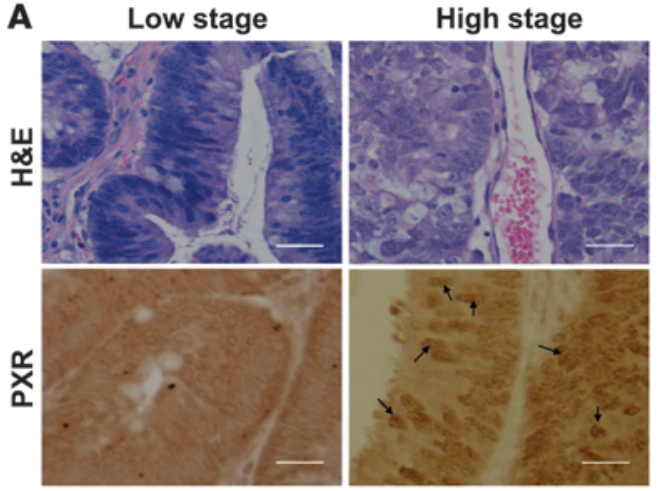

B
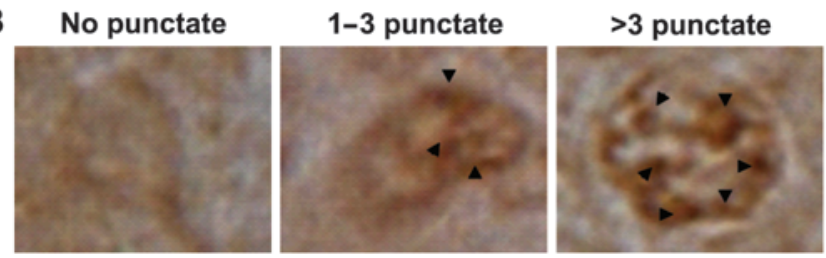

Figure 4

Nuclear PXR expression correlates with the clinical stage of primary human colon cancer tissue. (A) A representative primary colon tumor stained with $\mathrm{H} \& \mathrm{E}$ and antibody detecting PXR. Shown are the optimally stained histologic section for a low-stage tumor (AJCC stage I and II) and a high-stage tumor (AJCC stage III and IV). Black arrows indicate the intense punctuate nuclear staining for PXR. Scale bar: $50 \mu \mathrm{m}$. (B) Representative enlarged nucleus image (original magnification, $\times 63$ ), showing no nuclear punctate dots; 1-3 nuclear punctate dots; and more than 3 nuclear punctate dots. Arrowheads indicate punctate dots. (C) The PXR score (based on degree of nuclear staining with PXR antibody; for details, see Methods) is plotted based on the AJCC stage of the tumor (low, $n=45$; high, $n=45$ ). Data are presented as mean \pm SEM. ${ }^{*} P<0.0001$, comparing the 2 groups as indicated.

to downstream activation of ERK1/2 (Figure 3E). Activation of FRS $2 \alpha$ and ERK $1 / 2$ in response to rifampicin was suppressed by the FGF19 antibody 1A6, indicating that these effects are mediated by FGF19 (Figure 3F). Together, these data show that PXR activation in human colon cancers induces proliferation in vivo, which is mediated by enhanced FGF19 activity.

While we have provided firm evidence for a link between PXR activation and FGF19 signaling both in vitro and in vivo, the clinical relevance of PXR expression in solid tumors remains controversial. Indeed, different groups have used different methodologies to detect PXR expression in vivo, complicating interpretation of the data. In our preliminary immunostaining analysis of human colon cancer, we have qualitatively observed that tumors with intense PXR antibody staining tend to have discrete punctate staining largely within the nucleus (as has been observed for PXR activation in cell lines). Cytoplasmic staining is often diffuse and nonspecific. Therefore, to develop a robust criteria for the evaluation of PXR content in tumor specimens, we assigned scores based on finding 3 or more distinct and discrete punctate "dots" within the nucleus of more than $50 \%$ of tumor cells within one high-power field (HPF) of view and then averaged this across 10 random HPFs ( 1 , no nuclear punctate dots; $2,>50 \%$ cells with 1-3 nuclear punctate dots; $3,>50 \%$ of cells with $>3$ nuclear punctate dots; Figure 4, A and B). Figure 4A shows a typical low (American Joint Commission on Cancer [AJCC] stage I and II) and high (AJCC stage III and IV) clinical stage colon cancer stained with

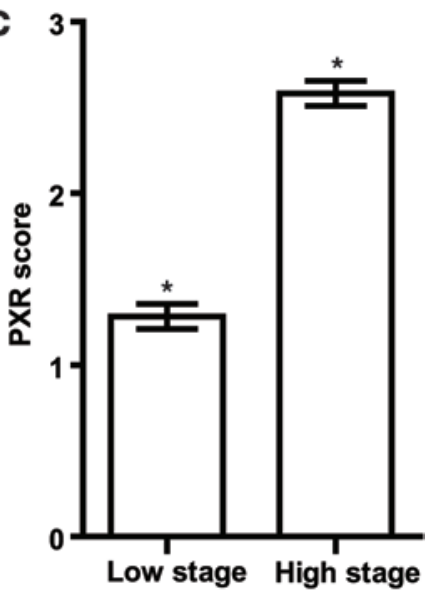

PXR antibody. Note that there is virtually no discrete PXR staining in the low-stage tumor, while the high-stage tumor shows discrete intense staining within the nuclei of tumor cells. Based on this assumption and arbitrary score threshold, we immunostained 90 randomly selected colon tumor specimens from our clinical pathology and commercial database (for details see Methods). We ensured that there would be an approximate balance of about 45 cases each of low-stage (associated with $\sim 80 \%-95 \%$ survival rate) and high-stage ( $<60 \%$ survival rate) tumors (50).

Based on our staining criteria, high-stage tumors had significantly greater abundance of nuclear punctate PXR staining than low-stage tumors (Figure 4C). Nuclear punctate PXR staining is observed in vitro during PXR activation (51), and, therefore, our results suggest that both the abundance and activation state of PXR are important determinants of tumor stage (and reduced cancer survival). Together, these data indicate that PXR activation in human colon cancers is associated with high clinical stage.

FGF19 is a direct target of PXR in colon cancer tissues through cancer tissue-specific activation of DR 3 elements on its promoter. PXR is expressed in both the small and large bowel enterocytes (52); however, its expression profile within the crypt-villus axis is not clearly characterized. Hence, we examined PXR expression in Caco-2 cells, a commonly used cell model for the study of enterocyte differentiation in vitro, and in primary crypt and villus enterocytes. As shown in Figure 5A, PXR mRNA expression was induced in Caco-2 cells upon differentiation. Similarly, $P X R$ mRNA was expressed at higher levels in villus enterocytes compared with that in crypt enterocytes (Figure 5B). Consistent with this finding, there was increased immunostaining for PXR protein in colonic villus cell nuclei and cytoplasm compared with that in crypt cells (Figure 5C). Thus, PXR expression is low in crypt enterocytes, and, consequently, crypt cells are much less responsive to PXR activation than villus cells. As shown in Figure 5D, induction of Fgf15 (the murine ortholog of human FGF19) expression by PXR activation in murine enterocytes was much less pronounced in crypt cells than in villus cells. Since nonneoplastic enterocytes (i.e., crypt cells) express much lower levels of PXR than colon cancer cells (specifically those with high clinical stage), we reasoned that the difference in PXR expression might account for why colon cancer cells induce FGF19 through PXR, leading to cell proliferation, migration, and metastasis. To this end, we tested whether forced expression of PXR in a human intestinal epithelial crypt cell (HIEC) line, which has growth and replication permissive properties, can confer upon these cells the ability to respond 
A

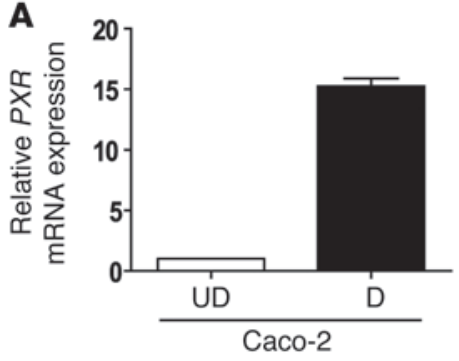

C
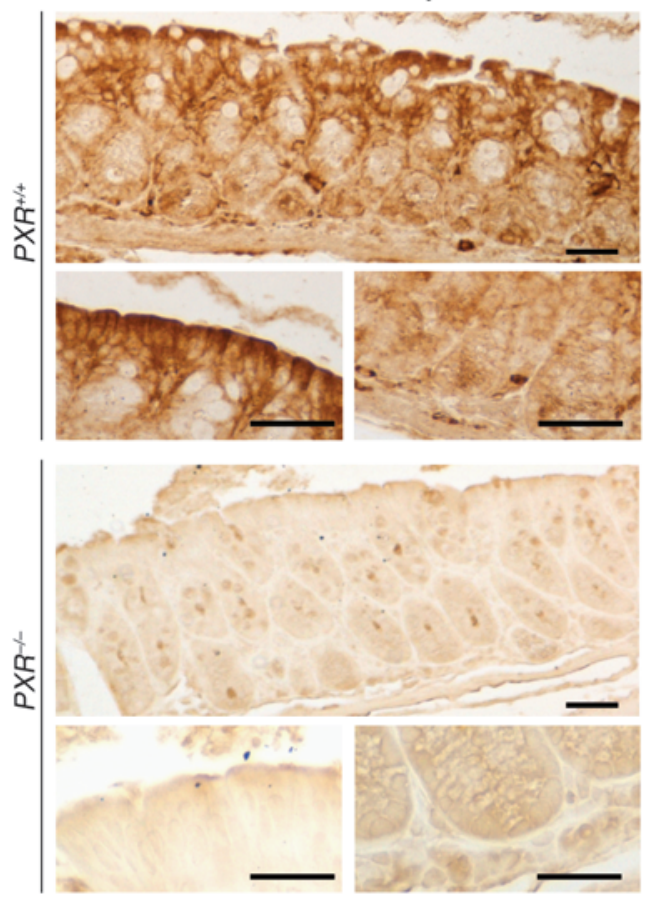

B

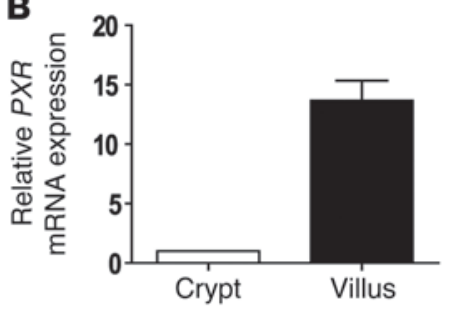

gG
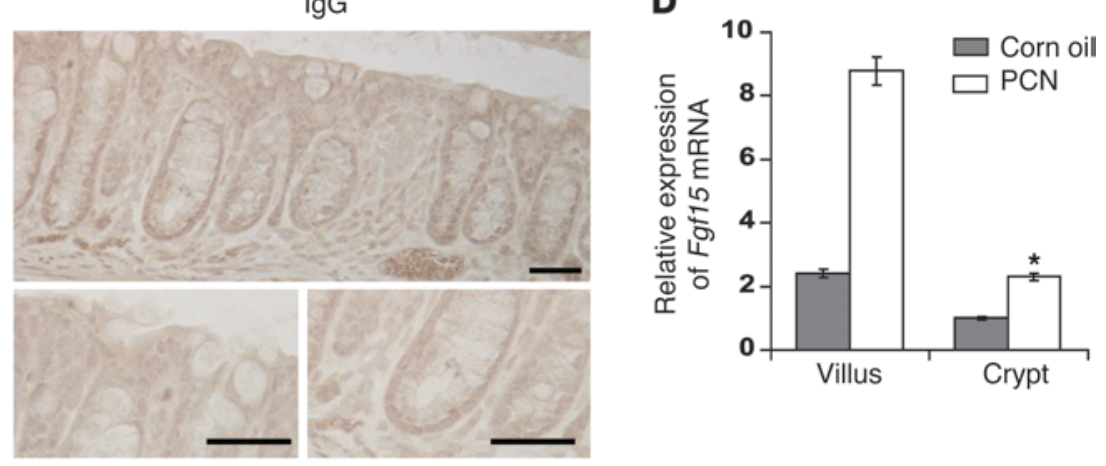

\section{Figure 5}

Expression of PXR and Fgf15 in enterocytes. Real-time QPCR for PXR in (A) Caco-2 and (B) murine enterocytes. Gene expression changes were calculated using the comparative Ct method, with $\beta$-actin as the reference gene and Caco-2 (undifferentiated [UD] cells) or crypt enterocytes as the calibrator, respectively. D, differentiated cells. (C) Immunohistochemical staining of PXR in large intestines (descending colon) from $P X R^{+/+}$and $P X R^{-/-}$mice. Apical villus cells with intense (dark brown) staining of PXR (diffuse cytoplasmic and punctate nuclear). Scale bar: 50 um. IgG, nonspecific antibody control. (D) Real-time QPCR for Fgf15 (murine ortholog of human FGF19) from murine villus and crypt cell total RNA. The mice had been treated with either PCN (150 mg/kg IP) or corn oil (PCN vehicle) for 3 consecutive days. Two-thirds of the proximal intestines were isolated, and cell fractions were obtained as described in Methods. Gene expression changes were calculated using comparative Ct method, with $\beta$-actin as the reference gene and crypt cells as the calibrator ( $n=2$ per group). (A, B, and D) $n=3$ in triplicate. Data are presented as mean \pm SEM.

to PXR activation in a similar way to that of LS174T colon cancer cells. As shown in Figure 6B, HIECs overexpressing PXR protein at levels comparable to those detected in LS174T cells (Figure 6, A and B; HIEC-PXR) exhibited increased proliferation upon addition of rifampicin (Figure 6C). These results were similar to those observed in other "immortalized" primary colonocytes, human colonic epithelial cells (HCECs) 1CT and 2CT (Supplemental Figure 9, A-C). In contrast to its effect on LS174T cells, however, rifampicin failed to induce FGF19 expression in HIEC-PXR cells (Figure 6, D and E), whereas it induced expression of the MDR1 gene, another known target gene of PXR (Figure 6E). Similarly, FGF19 was undetectable in rifampicin-treated HCECs (Ct values are undetermined). As expected, rifampicin had no effect on the expression of FGF19 in parental HIECs (data not shown). Notably, FGF19 also had minimal effects on HIEC and HCEC (1CT and 2CT) proliferation in vitro (Supplemental Figure 9, D-F). To investigate whether any differential DNA methylation pattern within the FGF19 promoter existed between LS174T cells and HIECs, we used the bisulfite conversion method of detecting DNA methylation and examined a 2-kb region upstream of the FGF19 promoter initiation start site. We did not find any significant difference between LS174T cells and HIECs in DNA methylation profile across this 2-kb region of the FGF19 promoter (data not shown).

To explore further why PXR activation in HIECs does not induce FGF19 expression, we examined whether PXR can bind to the FGF19 promoter in HIECs. In parallel, we analyzed PXR 
A

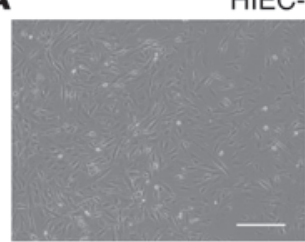

Phase contrast

C

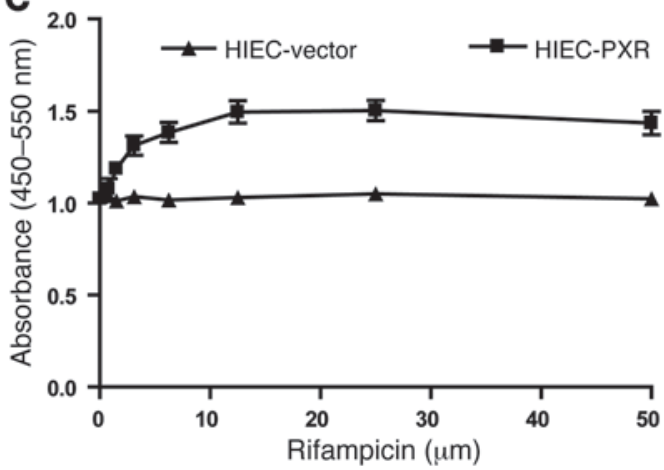

HIEC-vector

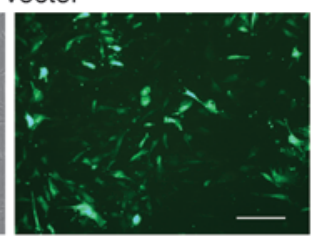

GFP

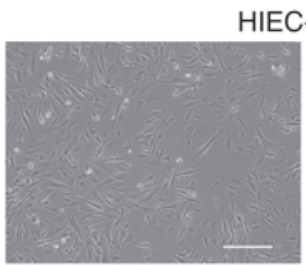

Phase contrast
HIEC-PXR

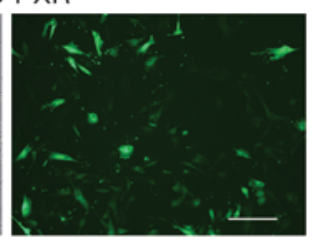

GFP
D

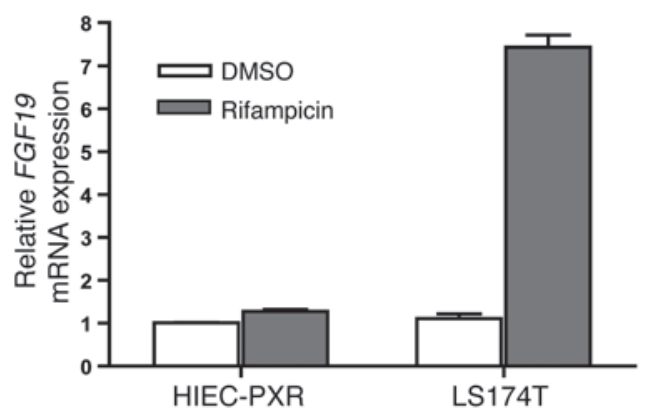

B

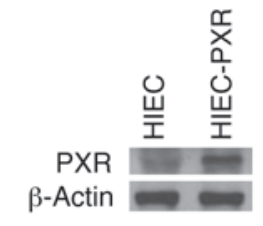

E

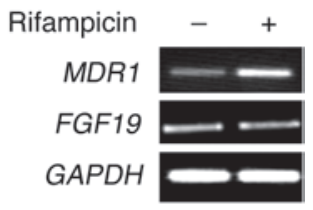

\section{Figure 6}

PXR activation induces FGF19 and promoter activity in a cell-type specific manner. (A) HIECs transfected with pEGFPC1 vector (HIEC) or pEGFPC1-hPXR (HIEC-PXR). Images were captured under appropriate filters (GFP and phase contrast). Scale bar: $100 \mu \mathrm{m}$. (B) Representative PXR immunoblot of pooled HIEC nuclear extract $(200 \mu \mathrm{g} / \mathrm{lane})$ from HIEC cells transfected with either pEGFPC1 vector (HIEC) or pEGFPC1-hPXR (HIEC-PXR). (C) Proliferation (BrdU) assay of HIEC-PXR cells and HIECs that had been treated for 48 hours with either rifampicin (0-50 $\mu \mathrm{M})$ or vehicle (0.2\% DMSO). The data is shown as absolute absorbance values (dual spectra 450-550 nm). (D) Real-time QPCR for FGF19 mRNA expression in LS174T and HIEC-PXR cells after cell stimulation with either rifampicin $(10 \mu \mathrm{M})$ or $0.2 \%$ DMSO for 48 hours $(n=3$ in quadruplicates). Gene expression changes were calculated using the comparative Ct method, with $\beta$-actin as the reference gene and DMSO-treated HIEC-PXR cells as the calibrator. (E) Semiquantitative RT-PCR for FGF19, MDR1, and GAPDH. HIEC-PXR cells had been stimulated for 48 hours with either rifampicin $(10 \mu \mathrm{M})$ or $0.2 \%$ DMSO (vehicle). Data are presented as mean \pm SEM.

binding to the FGF19 promoter in LS174T cells, and, as a control, we also analyzed PXR interaction with the CYP3A4 promoter in LS174T cells. Using ChIP, we detected binding of PXR to the FGF19 promoter in HIECs (Figure 7A). HIECs were transfected with an expression vector for PXR, so that PXR protein was expressed at levels sufficiently high to be able to immunoprecipitate the protein with currently available PXR antibodies. As expected, PXR also bound to the FGF19 promoter in LS174T cells (Figure 7B). We mapped PXR binding to DR3 and everted repeat 6 (ER6) elements on the FGF19 promoter, located within -85 bp from the transcription start site (Supplemental Figure 9), which had been identified as a prime candidate for PXR binding in a previous FGF19 promoter analysis $(21,22)$. Together, the data indicate that while PXR binds to the endogenous FGF19 promoter in both neoplastic and nonneoplastic colon epithelial cells, it only activates the promoter in neoplastic cells.

To further validate this finding, we examined whether PXR activation led to recruitment of RNA polymerase II (Pol II) to the FGF19 promoter in LS174T colon cancer cells. HIECs overexpressing PXR served as a negative control. As shown in Figure 7C, recruitment of Pol II to the FGF19 promoter was enhanced in LS174T cells in response to rifampicin. By contrast, as expected, rifampicin did not exhibit any effect on Pol II occupancy on the FGF19 promoter in HIECs overexpressing PXR (Figure 7C). These data imply that PXR activation leads to Pol II recruitment in the FGF19 promoter of LS174T colon cancer cells but not in the FGF19 promoter of nonneoplastic HIECs. To substantiate this further, we analyzed restric- tion enzyme site accessibility on the proximal FGF19 promoter in response to PXR activation. We chose the XhoI restriction site on the FGF19 promoter for these analyses, because it is located close to the Pol II binding site. We evaluated XhoI accessibility by amplifying a fragment of the FGF19 promoter containing the XhoI site before and after treatment of isolated cell nuclei with XhoI. For cell nuclei treated with XhoI for the indicated times (Figure 7D), the PCR product abundance decreased only in those promoters that were "accessible" to XhoI (open chromatin). As shown in Figure $7 \mathrm{D}$, stimulation of LS174T cells with rifampicin led to increased cleavage of the FGF19 promoter by XhoI. By contrast, rifampicin did not increase XhoI site accessibility on the FGF19 promoter in HIECs overexpressing PXR (Figure 7D). These data indicate that PXR activation leads to remodeling of the XhoI site on the proximal FGF19 promoter in LS174T colon cancer cells but not in nonneoplastic HIECs. Together, the findings suggest that PXR activates the FGF19 promoter in a tumor tissue-specific manner. While PXR is bound to the FGF19 promoter in nontumor tissues, activation of FGF19 transcription requires chromatin accessibility to enable Pol II recruitment, and the mechanisms governing this promoter accessibility appear to be tumor-tissue specific.

To test whether PXR could differentially transactivate a heterologous proximal FGF19 promoter in HIECs and LS174T cells, we cotransfected HIECs and LS174T cells with an approximately 2.2-kb FGF19 reporter construct and an expression plasmid encoding PXR. As shown in Figure 7E, in LS174T cells, rifampicin increased FGF19 promoter activity by almost 2 fold compared with unstimulated cells, 
A HIEC-PXR
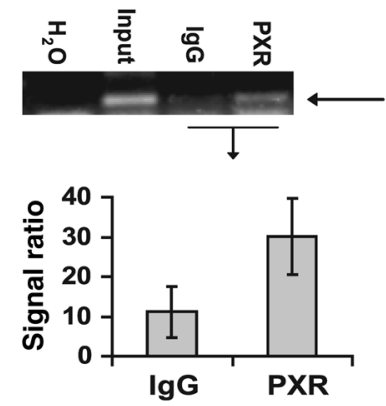

C

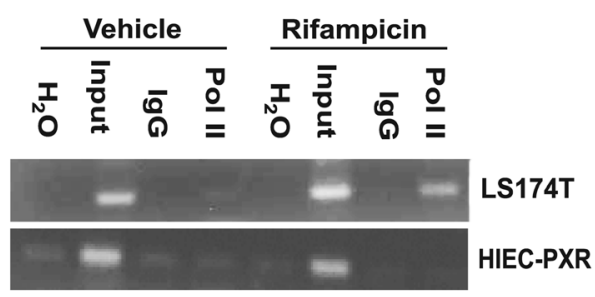

E

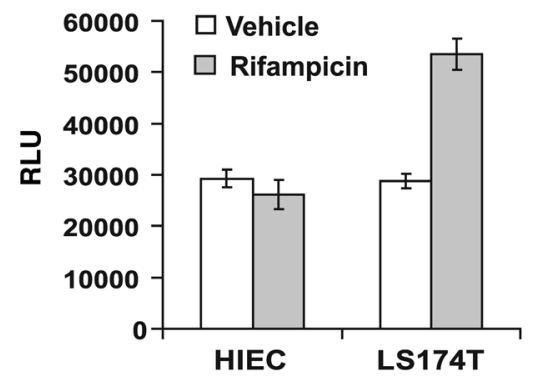

B

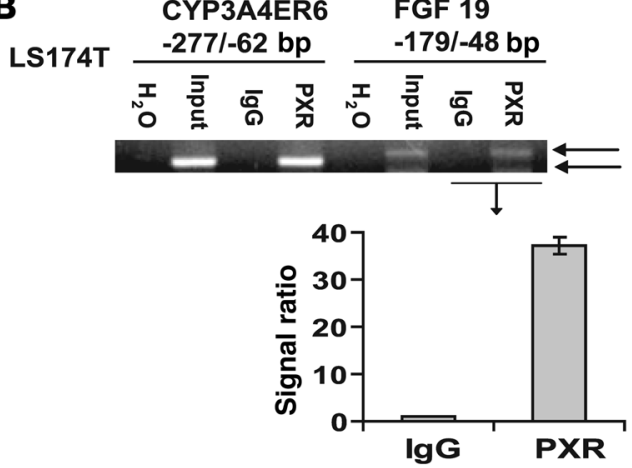

D

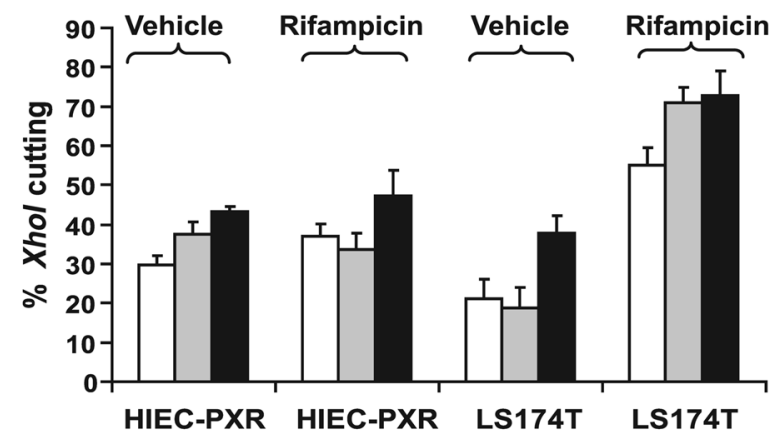

F

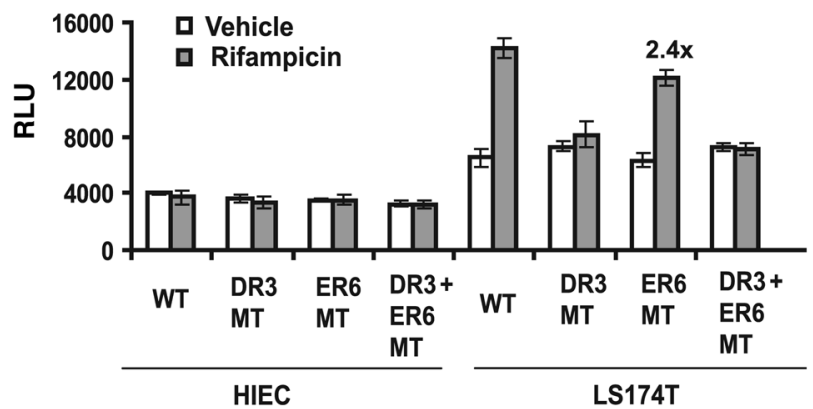

Figure 7

PXR binds to the endogenous FGF19 promoter and recruits RNA Pol II in LS174T cells. ChIP analysis of PXR in (A) HIEC-PXR and (B) LS174T cells. The ChIP analysis was performed 5 separate times (each time in duplicate) for QPCR and plotted as a signal ratio between the PXR and IgG lane (FGF19) as illustrated. (C) Pol II bound to endogenous FGF19 promoter in LS174T cells and HIECs overexpressing PXR (HIEC-PXR). (D) Xhol chromatin accessibility assay by real-time PCR (CHART-QPCR) was performed using cell nuclei from rifampicin- or vehicle-treated LS174T and HIEC-PXR cells (for primer sequence details, see Supplemental Table 1). The nuclei were treated with Xhol for 30 to 120 minutes. The Xhol accessibility was expressed as a percentage of the uncut DNA and is plotted against the time of Xhol digestion. All experiments were repeated at least 3 independent times, each in triplicate. CHART-QPCR assays were performed 4 independent times, with 6 repeats per assay point. (E) PXR transactivation assay was performed in LS174T cells and HIECs using the -2,216-bp FGF19 reporter ( $n=3$ in triplicate). (F) The PXR transactivation assay was performed in LS174T cells and HIECs using FGF19 promoter (-2,216-bp) wild-type, DR3 mutant, and/or ER6 mutant reporter constructs $(n=3$ in triplicate). Data are presented as mean \pm SEM $( \pm$ SD is shown for ChIP QPCR data). DMSO $(0.2 \%)$ was the vehicle for all in vitro experiments. "2.4x" indicates a 2.4 fold change compared with that of vehicle.

whereas in HIECs, rifampicin treatment had no effect on FGF19 promoter activity. To determine whether this differential activation of the approximately 2.2-kb FGF19 reporter was due to differential recognition and activation of PXR DNA binding elements, we cotransfected HIECs and LS174T cells with an approximately 2.2-kb FGF19 reporter or its DR3 and/or ER6 mutants (as shown in Figure 7F) and a plasmid encoding PXR. We selected mutants of the DR3 and ER6 elements within $-2.2 \mathrm{~kb}$ of the FGF19 promoter sequence for this transactiva- tion assay, because both elements are present in the proximal FGF19 promoter, PXR can bind to both elements, and mutants of DR3 and/ or ER6 are unable to bind to PXR (Supplemental Figure 10 and refs. 21, 22). As shown in Figure 7F, rifampicin induced approximately 2.4fold activation of DR3 elements in LS174T cells but did not activate the DR3 elements in HIEC-PXR cells. Surprisingly, rifampicin did not induce ER6 promoter activity in either LS174T or HIEC-PXR cells, even though PXR binds to this element in vitro (Supplemental Figure 10, 
for oligonucleotide sequence details, see Supplemental Table 2). Together, these data indicate that PXR activates the FGF19 promoter, and induces FGF19 expression, in colon cancer cells but not in nonneoplastic enterocytes. The selective induction of FGF19 in colon cancer cells and not in enterocytes is likely due to the cell-type specific ability of PXR to transactivate DR3 elements. Notably, the MDR1 promoter, which is activated by PXR in both HIECs and LS174T cells, contains DR4 and ER6/DR4 elements, which indicates that other PXR response elements may be activated in both cell types (53).

\section{Discussion}

In this study, we show that increased cancer cell malignant phenotype (increased cell proliferation, migration, and metastasis) in response to PXR activation by drugs is mediated through activation of FGF19 gene expression. Shih and Wistuba et al. have shown previously that PXR agonists induce activation of the FGF19 promoter (21, 22). Here, we map the PXR binding site on the FGF19 promoter and demonstrate that activation of PXR in cancer cells but not in nonneoplastic cells leads to chromatin remodeling, followed by Pol II recruitment at the FGF19 promoter. Since DR3 elements are not activated by PXR in "normal" colon cells, we surmise that there is deficient recruitment of specific cofactors engaging PXR on these elements. Together, our data provide an explanation for why FGF19 expression is specifically induced only in cancer cells in response to PXR activation.

PXR links the malignant phenotype with drug resistance, that is, PXR drives both processes. Since there are several environmental xenogens that can activate PXR at clinically relevant concentrations in humans, the implications that the environment plays a part in tumor recurrence through PXR are provocative and warrant further investigation.

The present study linking PXR activation to induction of FGF19 expression provides what we believe to be a novel avenue for cancer drug development. Indeed, in a sampling of 4 of our human colon tumors, there is detectable expression of FGFR1-FGFR4 and $\beta$ klotho (KLM) mRNA (Supplemental Figure 11). This raises the possibility that the FGF19/FGFR/ $\beta$ klotho pathway can be selectively targeted to reduce tumor proliferation and drug resistance in the context of chemotherapy. Presently, there is already one FGF19 antibody in development that shows good activity against colon cancer xenografts (24).

Our data are consistent with previous observations that PXR is antiapoptotic in colon (e.g., upregulates antiapoptotic BAG3, BIRC2, and MCL-1, while downregulating proapoptotic BAK1 and p53) (15, $54)$ and ovarian cancer $(20,55)$ and generally support the notion that PXR is associated with protection from xenobiotic damage (e.g., cellular drug resistance) $(54,56-59)$. However our observations are in stark contrast to recent observations that PXR induces apoptosis in colon (60) and breast cancer (18) and predicts for significantly improved survival in esophageal cancer (61). The discrepancy is likely due to methodological differences. For example, there is no criterion established thus far as to what constitutes high nuclear PXR expression (and activation). In this study, we defined objective criteria for nuclear expression of activated PXR, and, based on it, we found that PXR is associated with high rather than low clinically staged tumors. This has now been confirmed as a prognostic marker in ovarian cancer, Barrett's dysplasia, and other cancers $(55,62-64)$. These observations and our current results are consistent with an established role for PXR in inducing drug resistance in a variety of tumor types (19). Indeed, despite the modest differences we observed in the independent assays for proliferation, migration, and metastases in vitro and in vivo, the net effect may impact malignant potential that is clinically significant (60). Importantly, our PXR knockdown studies clearly show that induction of a malignant phenotype both in vitro and in vivo is specifically mediated by PXR. We have shown that our PXR knockdown clones are specific to PXR by mutant PXR rescue experiments that indeed rescue back lost phenotype. Additionally, we have extended our observations to multiple colon cell lines and primary human tumors studied in vitro and in vivo. Finally, we provide a mechanism which we believe to be novel underlying growth induction by PXR activation in colon cancer and demonstrate that this mechanism is specific to colon cancer cells as opposed to nonneoplastic colon crypt enterocytes. Our results have broad reaching implications that may mechanistically tie environmental xenogen exposure with cancer drug therapy response and survival.

\section{Methods}

Cell lines, tumor tissues, and reagents. Cell culture media and immunohistochemistry and PCR reagents were from Invitrogen by Life Technologies, unless indicated otherwise. (For more details, see Supplemental Methods.)

Plasmids construction and mutagenesis. The FGF19 promoter region encompassing the sequence between bases $-1,954$ and +244 (2,216 bp) was amplified by PCR from FGF19 BAC clone (RP-11-30016) using forward primer (XhoI site), 5'-CCCCTCGAGTCAACACCTTCATGAGTGCTACATC-3', and reverse primer (HindIII site), 5'-CCCAAGCTTGAGCATTTCTTATCGGGATTGCATC-3'. Digested PCR fragments were cloned into pGL4.10-luc vector (Promega). Site-directed mutagenesis of the DR3, ER6, and DR3/ER6 elements of FGF19 promoter construct was performed using the Quick-Change Site-Directed Mutagenesis Kit (Stratagene). The following primers were used for mutagenesis: FGF19 promoter DR3 mutant primer pairs (DR3 mutant [DR3 MT]), forward primer, 5'-TTAAGGGGACTTGCACTAAGGCCGAG-3', and reverse primer, 5'-CTCGGCCTTAGTGCAAGTCCCCTTAA-3'; and FGF19 promoter ER6 mutant primer pairs (ER6 MT), forward primer, 5'-TGGCCCCGGTACCCACGCCGAGTGCATC-3', and reverse primer, 5'-GATGCACTCGGCGTGGGTACCGGGGCCA-3'. Bold letters in the primer pairs indicate the mutated base pairs. (For construction of silent PXR mutants, see Supplemental Methods.)

Histology and immunohistochemistry. Tumor nodule sections from mice (liver, spleen, and tumor heterotransplants), primary human tumors, and mouse intestines were paraffin embedded and cut to 5 -micron thickness on uncoated slides. After dewaxing and rehydrating, the slides were placed in a sodium citrate solution, pH 6.0 (Vector Laboratories), for 20 minutes. Details regarding blocking and incubations with PXR and secondary antibody have been published previously (20). H\&E, Ki-67 (1:50), and CEA (1:50) staining were performed as described previously (65). The PXR score was determined by the number of nuclear punctuate dots: 1 , no nuclear punctuate dots; 2 , more than $50 \%$ of cells with 1 to 3 nuclear punctuate dots; and 3 , more than $50 \%$ of cells with more than 3 nuclear punctuate dots across 10 random HPFs.

In vivo experiments. All animal and tumor acquisition experiments were approved by the Albert Einstein College of Medicine IACUC and Committee on Clinical Investigations (CCI) (IACUC no. 20070715 and 20100711; CCI no. 2007-554), respectively. (For in vivo experiments details, see Supplemental Methods.)

In vivo liver metastasis. The in vivo liver metastasis model was developed as previously published $(47,48)$. (For experimental details, see Supplemental Methods.)

Lentivirus-based shRNA knockdown systems. For PXR shRNA lentiviral plasmid design and construction, we used a protocol published by our collaborator (Liang Zhu) (66). (For details on both FGF19 and PXR shRNA, see Supplemental Methods.) 
Isolation of murine small bowel enterocyte fractions. We used the Weiser method to isolate pure crypt and villus fractions from the small bowel of C57BL/6 mice $(67,68)$.

Electrophoretic mobility shift assay. The gel shift assays were performed as described previously (40). (For details, see Supplemental Methods.)

Luciferase assay. LS174T and HIECs were seeded into 24-well plates. After 24 hours, cells were transiently transfected with LacZ plasmid and/or pSG5-PXR and FGF19 promoter (2,216 bp) wild-type, DR3 mutant, ER6 mutant, or DR3 plus ER6 mutant reporter constructs, and luciferase assay was performed as previously published $(9,40)$.

Cell proliferation assays (doubling time), transwell migration assay, $R N A$ preparation and semiquantitative $R T-P C R$, real-time quantitative $P C R$, chromatin accessibility by real-time PCR assay, immunoblotting, DNA methylation, ChIP assay, and human tumor heterotransplant experiments. See Supplemental Methods.

Statistics. Student's $t$ test (2-tailed) was used to analyze differences between 2 groups. Trend analyses between 3 or more groups were per- formed using ANOVA test. All the results were expressed as mean \pm SEM. $P$ values of less than 0.05 were considered to be significant. All analyses were performed using GraphPad software.

\section{Acknowledgments}

This work was supported in part by a grant from the Damon Runyon Cancer Research Foundation (CI: 15-02 to S. Mani) and grants from the NIH (CA127231 to S. Mani, DE13686 to M. Mohammadi, and AG019712 to M. Kuro-o).

Received for publication September 16, 2010, and accepted in revised form May 18, 2011.

Address correspondence to: Sridhar Mani, Albert Einstein College of Medicine, 1300 Morris Park Ave., Chanin 302D-1, New York, New York 10461, USA. Phone: 718.430.2871; Fax: 718.904.2830; E-mail: sridhar.mani@einstein.yu.edu.
1. Pasini F, Pelosi G, De Manzoni G, Rosti G. Highdose chemotherapy in small cell lung cancer. Tumori. 2002;88(3):179-186.

2. Ledermann JA. Lessons learned from a decade of clinical trials of high-dose chemotherapy in ovarian cancer. Int J Gynecol Cancer. 2008;18 suppl 1:53-58.

3. Vogl DT, Stadtmauer EA. High-dose chemotherapy and autologous hematopoietic stem cell transplantation for metastatic breast cancer: a therapy whose time has passed. Bone Marrow Transplant. 2006;37(11):985-987.

4. Seidman AD. Sequential single-agent chemotherapy for metastatic breast cancer: therapeutic nihilism or realism? J Clin Oncol. 2003;21(4):577-579.

5. Brabletz T, Jung A, Spaderna S, Hlubek F, Kirchner T. Opinion: migrating cancer stem cells - an integrated concept of malignant tumour progression. Nat Rev Cancer. 2005;5(9):744-749.

6. Blagosklonny MV. Cell immortality and hallmarks of cancer. Cell Cycle. 2003;2(4):296-299.

7. Dean M, Fojo T, Bates S. Tumour stem cells and drug resistance. Nat Rev Cancer. 2005;5(4):275-284.

8. Chawla A, Repa JJ, Evans RM, Mangelsdorf DJ. Nuclear receptors and lipid physiology: opening the X-files. Science. 2001;294(5548):1866-1870.

9. Kliewer SA, Goodwin B, Willson TM. The nuclear pregnane $\mathrm{X}$ receptor: a key regulator of xenobiotic metabolism. Endocr Rev. 2002;23(5):687-702.

10. Blumberg B, et al. SXR, a novel steroid and xenobiotic-sensing nuclear receptor. Genes Dev. 1998;12(20):3195-3205.

11. Mani S, et al. Activation of the steroid and xenobiotic receptor (human pregnane $\mathrm{X}$ receptor) by nontaxane microtubule-stabilizing agents. Clin Cancer Res. 2005;11(17):6359-6369.

12. Kliewer SA, et al. An orphan nuclear receptor activated by pregnanes defines a novel steroid signaling pathway. Cell. 1998;92(1):73-82.

13. Mikamo E, Harada S, Nishikawa J, Nishihara T. Endocrine disruptors induce cytochrome P450 by affecting transcriptional regulation via pregnane $\mathrm{X}$ receptor. Toxicol Appl Pharmacol. 2003;193(1):66-72.

14. Kortagere S, Krasowski MD, Reschly EJ, Venkatesh M, Mani S, Ekins S. Evaluation of computational docking to identify PXR agonists in the ToxCast(TM) database. Environ Health Perspect. 2010;118(10):1412-1417.

15. Zhou J, Liu M, Zhai Y, Xie W. The antiapoptotic role of pregnane $X$ receptor in human colon cancer cells. Mol Endocrinol. 2008;22(4):868-880.

16. Chen Y, Tang Y, Wang MT, Zeng S, Nie D. Human pregnane $X$ receptor and resistance to chemotherapy in prostate cancer. Cancer Res. 2007; 67(21):10361-10367.

17. Masuyama H, Nakatsukasa H, Takamoto N, Hira- matsu Y. Down-regulation of pregnane $\mathrm{X}$ receptor contributes to cell growth inhibition and apoptosis by anticancer agents in endometrial cancer cells. Mol Pharmacol. 2007;72(4):1045-1053.

18. Verma S, Tabb MM, Blumberg B. Activation of the steroid and xenobiotic receptor, SXR, induces apoptosis in breast cancer cells. BMC Cancer. 2009;9:3.

19. Biswas A, Mani S, Redinbo MR, Krasowski MD, Li H, Ekins S. Elucidating the 'Jekyll and Hyde' nature of PXR: the case for discovering antagonists or allosteric antagonists. Pharm Res. 2009;26(8):1807-1815.

20. Gupta D, et al. Expanding the roles for pregnane $X$ receptor in cancer: proliferation and drug resistance in ovarian cancer. Clin Cancer Res. 2008;14(17):5332-5340.

21. Shih DM, Kast-Woelbern HR, Wong J, Xia YR, Edwards PA, Lusis AJ. A role for FXR and human FGF19 in the repression of paraoxonase-1 gene expression by bile acids. J Lipid Res. 2006;47(2):384-392.

22. Wistuba W, Gnewuch C, Liebisch G, Schmitz G, Langmann T. Lithocholic acid induction of the FGF19 promoter in intestinal cells is mediated by PXR. World J Gastroenterol. 2007;13(31):4230-4235.

23. Schmidt DR, Mangelsdorf DJ. Nuclear receptors of the enteric tract: guarding the frontier. Nutr Rev. 2008;66(10 suppl 2):S88-S97.

24. Desnoyers LR, et al. Targeting FGF19 inhibits tumor growth in colon cancer xenograft and FGF19 transgenic hepatocellular carcinoma models. Oncogene. 2008;27(1):85-97.

25. Beenken A, Mohammadi M. The FGF family: biology, pathophysiology and therapy. Nat Rev Drug Discov. 2009;8(3):235-253.

26. Wu X, et al. Separating mitogenic and metabolic activities of fibroblast growth factor 19 (FGF19). Proc Natl Acad Sci U S A. 2010;107(32):14158-14163.

27. Taylor JGt, et al. Identification of FGFR4-activating mutations in human rhabdomyosarcomas that promote metastasis in xenotransplanted models. J Clin Invest. 2009;119(11):3395-3407.

28. Roidl A, Berger HJ, Kumar S, Bange J, Knyazev P, Ullrich A. Resistance to chemotherapy is associated with fibroblast growth factor receptor 4 up-regulation. Clin Cancer Res. 2009;15(6):2058-2066.

29. Meijer D, Sieuwerts AM, Look MP, van Agthoven T, Foekens JA, Dorssers LC. Fibroblast growth factor receptor 4 predicts failure on tamoxifen therapy in patients with recurrent breast cancer. Endocr Relat Cancer. 2008;15(1):101-111.

30. Soto AM, Sonnenschein C. Environmental causes of cancer: endocrine disruptors as carcinogens. Nat Rev Endocrinol. 2010;6(7):363-370.

31. LaPensee EW, Ben-Jonathan N. Novel roles of prolactin and estrogens in breast cancer: resis- tance to chemotherapy. Endocr Relat Cancer. 2010; 17(2):R91-R107.

32. LaPensee EW, LaPensee CR, Fox S, Schwemberger $\mathrm{S}$, Afton S, Ben-Jonathan N. Bisphenol A and estradiol are equipotent in antagonizing cisplatininduced cytotoxicity in breast cancer cells. Cancer Lett. 2010;290(2):167-173.

33. Dairkee SH, et al. Bisphenol A induces a profile of tumor aggressiveness in high-risk cells from breast cancer patients. Cancer Res. 2008;68(7):2076-2080.

34. Xu X, Dailey AB, Talbott EO, Ilacqua VA, Kearney G, Asal NR. Associations of serum concentrations of organochlorine pesticides with breast cancer and prostate cancer in U.S. adults. Environ Health Perspect. 2010;118(1):60-66.

35. Zhu H, et al. Environmental endocrine disruptors promote invasion and metastasis of SKN-SH human neuroblastoma cells. Oncol Rep. 2010;23(1):129-139.

36. Takeshita A, Inagaki K, Igarashi-Migitaka J, Ozawa Y, Koibuchi N. The endocrine disrupting chemical, diethylhexyl phthalate, activates MDR1 gene expression in human colon cancer LS174T cells. J Endocrinol. 2006;190(3):897-902.

37. Diamanti-Kandarakis E, et al. Endocrine-disrupting chemicals: an Endocrine Society scientific statement. Endocr Rev. 2009;30(4):293-342.

38. Garg BD, Kovacs K, Blascheck JA, Selye H. Ultrastructural changes induced by pregnenolone nitrile in the rat liver. J Pharm Pharmacol. 1970;22(11):872-873.

39. Dai G, He L, Bu P, Wan YJ. Pregnane X receptor is essential for normal progression of liver regeneration. Hepatology. 2008;47(4):1277-1287.

40. Huang $\mathrm{H}$, et al. Inhibition of drug metabolism by blocking the activation of nuclear receptors by ketoconazole. Oncogene. 2007;26(2):258-268.

41. Lehmann JM, McKee DD, Watson MA, Willson TM, Moore JT, Kliewer SA. The human orphan nuclear receptor PXR is activated by compounds that regulate CYP3A4 gene expression and cause drug interactions. J Clin Invest. 1998;102(5):1016-1023.

42. Gonzalez FJ. CYP3A4 and pregnane X receptor humanized mice. J Biochem Mol Toxicol. 2007;21(4):158-162.

43. Watkins RE, Noble SM, Redinbo MR. Structural insights into the promiscuity and function of the human pregnane X receptor. Curr Opin Drug Discov Devel. 2002;5(1):150-158.

44. Watkins RE, et al. The human nuclear xenobiotic receptor PXR: structural determinants of directed promiscuity. Science. 2001;292(5525):2329-2333.

45. Moore LB, et al. Orphan nuclear receptors constitutive androstane receptor and pregnane $\mathrm{X}$ receptor share xenobiotic and steroid ligands. J Biol Chem. 2000;275(20):15122-15127. 
46. Stover DR, Becker M, Liebetanz J, Lydon NB. Src phosphorylation of the epidermal growth factor receptor at novel sites mediates receptor interaction with Src and P85 alpha. J Biol Chem. 1995;270(26):15591-15597.

47. Xue KX, Gao J. [Study of liver metastasis formation and its mechanism using intrasplenic inoculation of cancer cells]. Zhonggno Yi Xue Ke Xue Yuan Xue Bao. 1987;9(3):217-222.

48. Thalheimer A, et al. The intraportal injection model: a practical animal model for hepatic metastases and tumor cell dissemination in human colon cancer. BMC Cancer. 2009;9:29.

49. Goetz R, et al. Isolated C-terminal tail of FGF23 alleviates hypophosphatemia by inhibiting FGF23FGFR-Klotho complex formation. Proc Natl Acad Sci US A. 2010;107(1):407-412.

50. Burke HB. Outcome prediction and the future of the TNM staging system. J Natl Cancer Inst. 2004;96(19):1408-1409.

51. Wang H, et al. The phytoestrogen coumestrol is a naturally occurring antagonist of the human pregnane X receptor. Mol Endocrinol. 2008; 22(4):838-857.

52. Shah YM, Ma X, Morimura K, Kim I, Gonzalez FJ. Pregnane $\mathrm{X}$ receptor activation ameliorates DSSinduced inflammatory bowel disease via inhibition of NF-kappaB target gene expression. Am J Physiol Gastrointest Liver Physiol. 2007;292(4):G1114-G1122.

53. Geick A, Eichelbaum M, Burk O. Nuclear receptor response elements mediate induction of intestinal MDR1 by rifampin. J Biol Chem. 2001;276(18):14581-14587.

54. Habano W, et al. Involvement of promoter methylation in the regulation of Pregnane $\mathrm{X}$ receptor in colon cancer cells. BMC Cancer. 2011;11:81.

55. Yue X, et al. Steroid and Xenobiotic Receptor (SXR) as a possible prognostic marker in epithelial ovarian cancer. Pathol Int. 2010;60(5):400-406.

56. Raynal C, et al. Pregnane X Receptor (PXR) expression in colorectal cancer cells restricts irinotecan chemosensitivity through enhanced SN-38 glucuronidation. Mol Cancer. 2010;9:46.

57. Jiang $\mathrm{H}$, et al. Association of pregnane $\mathrm{X}$ receptor with multidrug resistance-related protein 3 and its role in human colon cancer chemoresistance.J Gastrointest Surg. 2009;13(10):1831-1838.

58. Harmsen S, Meijerman I, Febus CL, Maas-Bakker RF, Beijnen JH, Schellens JH. PXR-mediated induction of P-glycoprotein by anticancer drugs in a human colon adenocarcinoma-derived cell line. Cancer Chemother Pharmacol. 2010;66(4):765-771.

59. Kodama S, Negishi M. Pregnane X Receptor PXR Activates the GADD45\{beta\} Gene, Eliciting the p38 MAPK Signal and Cell Migration.J Biol Chem. 2011;286(5):3570-3578.

60. Ouyang N, et al. Pregnane X receptor suppresses proliferation and tumourigenicity of colon cancer cells. Br J Cancer. 2010;102(12):1753-1761.

61. Takeyama D, et al. Steroid and xenobiotic receptor in human esophageal squamous cell carcinoma: a potent prognostic factor. Cancer Sci.
2010;101(2):543-549.

62. van de Winkel A, et al. Differential expression of the nuclear receptors farnesoid $X$ receptor (FXR) and pregnane $X$ receptor (PXR) for grading dysplasia in patients with Barrett's oesophagus. Histopathology. 2011;58(2):246-253.

63. Conde I, et al. Human pregnane X receptor is expressed in breast carcinomas, potential heterodimers formation between hPXR and RXR-alpha. BMC Cancer. 2008;8:174.

64. Rioja J, et al. Association of steroid and xenobiotic receptor (SXR) and multidrug resistance 1 (MDR1) gene expression with survival among patients with invasive bladder carcinoma. BJU Int. 2011;107(11):1833-1838.

65. Horig $\mathrm{H}$, et al. A new mouse model for evaluating the immunotherapy of human colorectal cancer. Cancer Res. 2001;61(23):8520-8526.

66. Sun D, Melegari M, Sridhar S, Rogler CE, Zhu L. Multi-miRNA hairpin method that improves gene knockdown efficiency and provides linked multigene knockdown. Biotechniques. 2006;41(1):59-63.

67. Ferraris RP, Villenas SA, Diamond J. Regulation of brush-border enzyme activities and enterocyte migration rates in mouse small intestine. Am J Physiol. 1992;262(6 pt 1):G1047-G1059.

68. Weiser MM. Intestinal epithelial cell surface membrane glycoprotein synthesis. II. Glycosyltransferases and endogenous acceptors of the undifferentiated cell surface membrane. J Biol Chem. 1973;248(7):2542-2548. 